\title{
In Sickness and in Health: The Co-Regulation of Inflammation and Social Behavior
}

\author{
Naomi I Eisenberger ${ }^{\star, 1}$, Mona Moieni ${ }^{1}$, Tristen K Inagaki ${ }^{2}$, Keely A Muscatell ${ }^{3}$ and Michael R Irwin ${ }^{4}$ \\ ${ }^{1}$ Department of Psychology, University of California, Los Angeles, CA, USA; ${ }^{2}$ Department of Psychology, University of \\ Pittsburgh, Pittsburgh, PA, USA; ${ }^{3}$ Department of Psychology, University of North Carolina-Chapel Hill, Chapel Hill, NC, USA; \\ ${ }^{4}$ Department of Psychiatry and Biobehavioral Sciences and Cousins Center for Psychoneuroimmunology, University of \\ California, Los Angeles, CA, USA
}

Although it has commonly been assumed that the immune system and the processes that govern social behavior are separate, non-communicating entities, research over the past several decades suggests otherwise. Considerable evidence now shows that inflammatory processes and social behavior are actually powerful regulators of one another. This review first summarizes evidence that inflammatory processes regulate social behavior, leading to characteristic changes that may help an individual navigate the social environment during times of sickness. Specifically, this review shows that inflammation: (1) increases threatrelated neural sensitivity to negative social experiences (eg, rejection, negative social feedback), presumably to enhance sensitivity to threats to well-being or safety in order to avoid them and (2) enhances reward-related neural sensitivity to positive social experiences (eg, viewing close others and receiving positive social feedback), presumably to increase approach-related motivation towards others who might provide support and care during sickness. Next, this review summarizes evidence showing that social behavior also regulates aspects of inflammatory activity, preparing the body for situations in which wounding and infection may be more likely (social isolation). Here, we review research showing: (1) that exposure to social stressors increases proinflammatory activity, (2) that individuals who are more socially isolated (ie, lonely) show increased proinflammatory activity, and (3) that individuals who are more socially isolated show increased proinflammatory activity in response to an inflammatory challenge or social stressor. The implications of the co-regulation of inflammation and social behavior are discussed.

Neuropsychopharmacology Reviews (2017) 42, 242-253; doi:I0.1038/npp.2016.141; published online I4 September 2016

\section{INTRODUCTION}

Although inflammatory activity is known primarily for its role as the body's first line of defense against tissue damage and microbial infection, research over the past several decades has revealed that inflammatory activity is also a powerful organizer of behavior. For instance, proinflammatory cytokines, one of the key chemical messengers of the immune system, not only orchestrate peripheral inflammatory responses to prevent infection (such as in response to a microbial antigen), but these cytokines also signal the brain to alter behavior. Specifically, through a variety of mechanisms, including activation of afferent vagal nerves (ie, proinflammatory cytokines bind to cells of the vagal paraganglia and activate

${ }^{*}$ Correspondence: $\mathrm{Dr} \mathrm{Nl}$ Eisenberger, Department of Psychology, University of California, 1285 Franz Hall, Box 951563, Los Angeles, CA 90095-1563, USA, Tel: +1 310267 5196, Fax: +1 310206 5895, E-mail: neisenbe@ucla.edu

Received 8 April 2016; revised 16 June 2016; accepted 18 July 2016; accepted article preview online 2 August 2016 the vagal nerve; Goehler et al, 1997) and transport through the blood-brain barrier (BBB; Dantzer et al, 2008; Maier and Watkins, 1998), cytokines can signal the brain to invoke a constellation of behaviors known as sickness behaviors (Hart, 1988; Dantzer, 2001; Kelley et al, 2003; Quan and Banks, 2007). These behaviors include loss of appetite, sleepiness, social withdrawal, fatigue, increased pain, and anhedonia, and are conceptualized as a coordinated motivational response thought to facilitate recuperation and recovery from illness and disease (Dantzer and Kelley, 2007; Hart, 1988).

However, more recently, research has begun to reveal that inflammation is also a particularly powerful organizer of social behavior (Eisenberger et al, 2009, 2010b; Hennessy et al, 2014; Moieni et al, 2015b; Moieni et al, 2015c). Although this is somewhat surprising, given the seeming disconnect between sickness on the one hand and social behavior on the other, the relationship between inflammation and social behavior may provide a survival advantage. Being in a 'sick state' or a state of heightened inflammation puts a social organism in a particularly vulnerable situation and thus sensitivity to the 
social world may need to be altered to navigate this more vulnerable situation. Navigating sickness for a social species may be accomplished by: (1) increasing sensitivity to threatening social experiences (Eisenberger et al, 2009; Inagaki et al, 2012; Muscatell et al, 2016) in order to better identify and avoid threats to well-being while in this vulnerable state and (2) increasing approach-related behavior towards close others (Inagaki et al, 2015) or other individuals who might be able to provide support (Muscatell et al, 2016) in order to recruit help and care in order to facilitate recovery from sickness (Figure 1, left side). Here, we review evidence from both animal and human research showing that states of heightened inflammation lead to several important changes in social behavior. We will also discuss how these inflammatoryinduced alterations in social behavior may ultimately help to explain the relationship between heightened levels of inflammation and the presence of certain psychiatric disorders that involve altered social sensitivities (eg, depression).

Finally, in addition to inflammation being a powerful organizer of social behavior, social behavior may also have a powerful effect on regulating the immune system. Given the importance of social connection for human survival and the fact that social disconnection severely compromises survival-increasing risk of predation, wounding, and infection-recent theories have suggested that the immune system may respond to various forms of social disconnection by upregulating proinflammatory response genes to prepare the body for these more vulnerable situations (Cole et al, 2007; Eisenberger and Cole, 2012). For instance, to the extent that an individual is socially disconnected (ostracized and excluded), that individual faces a greater risk of wounding and infection as a function of greater vulnerability to predation or attack by hostile conspecifics without protection from others. To anticipate and protect against these possibilities, the body may respond to these potential threats by upregulating proinflammatory response genes to prepare the body for situations in which wounding is more likely. Along these lines, we review evidence showing that exposure to socially threatening situations can increase inflammatory activity, and that individuals who are lonely or socially disconnected tend to show higher levels of proinflammatory activation (Cole et al, 2007; Moieni et al, 2015a; Figure 1, right side).

\section{PART I: INFLAMMATION AS AN ORGANIZER OF SOCIAL BEHAVIOR}

Nearly all discussions of inflammatory-induced sickness behavior include social withdrawal as one of the key symptoms: animals show reduced social exploration and socially withdraw when sick in order to promote recovery and recuperation from illness or infection (Kelley et al, 2003). However, even though these changes in social behavior are an accepted part of the sickness response, very little research has carefully interrogated the social behavioral consequences of inflammation. Given that heightened inflammation puts organisms in a vulnerable situation, needing to stay away

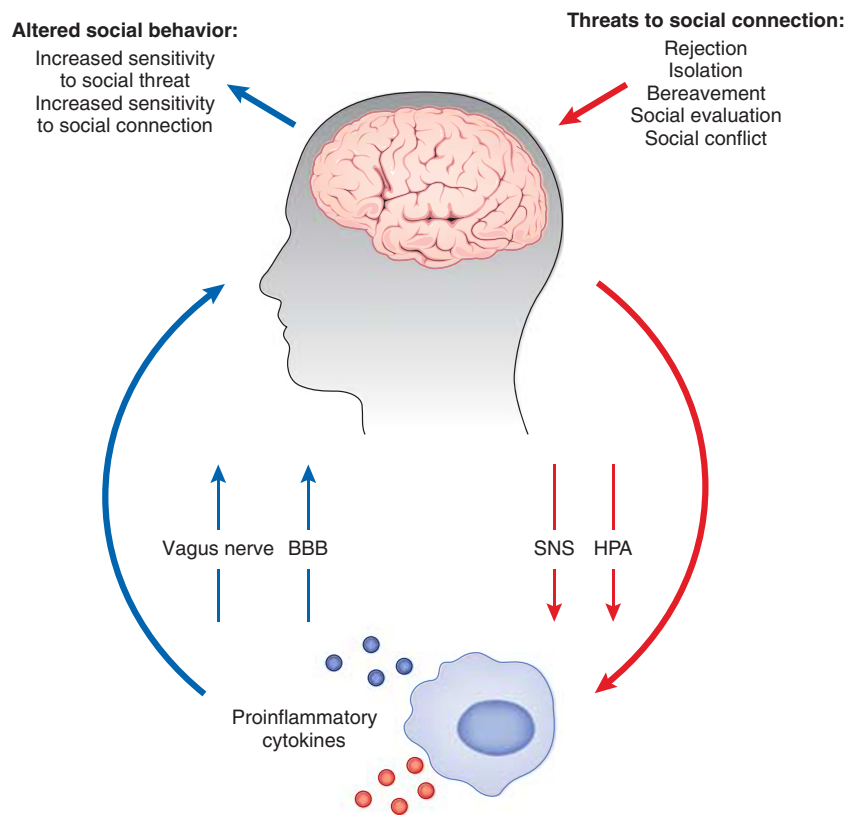

Figure 1. The co-regulation of inflammation and social behavior. The left side of the panel shows that proinflammatory cytokines, through acting on the vagus nerve or the BBB (among other mechanisms) can signal the brain to alter neural sensitivity to the social environment, leading to increases in sensitivity to social threat and social connection. The right side of the panel shows that experiences that threaten social connection (eg, rejection and isolation) can activate certain systems (SNS, sympathetic nervous system; HPA, hypothalamic-pituitary-adrenocortical axis), which can ultimately upregulate proinflammatory activity.

from unfriendly strangers (due to possible harm), but also needing additional care from close others, it is important to examine how inflammation alters social behavior in specific ways. Here, we review research examining the ways in which inflammation alters sensitivity to different forms of social experience in both animals and humans.

\section{Effects of Inflammation on Social Behavior in Animals}

Interestingly, the commonly accepted notion that inflammation induces social withdrawal is based on studies of adult rats showing that systemic administration of lipopolysaccharide (LPS), a bacterial agent that triggers an inflammatory response, reduces their interest in exploring novel juvenile rats (Bluthé et al, 1992; Bluthé et al, 1994). Although this is an important piece of data showing that inflammation can alter social behavior, it represents only one aspect of sociality that could be explored. Importantly, when the type of social behavior is broadened beyond the exploration of novel social targets, we find that social withdrawal is not an invariant response to sickness (Hennessy et al, 2014). Instead, the effect of inflammation on social behavior depends on the particular situation and whether social approach or social avoidance behavior is more adaptive in that situation.

For instance, research has demonstrated that when an animal is interacting with a familiar other, as opposed to a 
stranger, inflammation can actually increase certain forms of social behavior. Hence, in rats, in addition to reducing active social behavior with an unfamiliar conspecific, LPS can also lead to more huddling with familiar cagemates (Yee and Prendergast, 2010). Similarly, juvenile rhesus monkeys injected with low-dose LPS spent more time in close proximity with and clinging to a familiar cage-mate than did vehicle-injected controls (Willette et al, 2007). Finally, among prairie voles, females injected with LPS (compared with those injected with saline) actually established preferences for a male partner more rapidly (Bilbo et al, 1999). Thus, although inflammation can decrease social approach towards unfamiliar others (Bluthé et al, 1992; Bluthé et al, 1994), it can simultaneously increase social approach behavior towards familiar others, potentially in order to obtain much needed care from them (eg, protection, comfort, and nourishment).

Thus, a closer look at animal models suggests that inflammation's effect on social behavior is much more complex than what has commonly been assumed-namely, that inflammation induces social withdrawal. More specifically, how inflammation alters social behavior may depend, in part, on the competing motivational priorities of the sick individual. In some cases, it may be more beneficial for survival to remain in close contact with familiar others, rather than withdrawing, in order to garner care and support during sickness. Not surprisingly then, it is interesting to note that the social consequences of inflammation that conflict most with the simple social withdrawal account are typically observed in the most social species (prairie voles and rhesus monkeys; Hennessy et al, 2014). Indeed, research in humans builds on these findings by showing that inflammation alters social experience in complex ways, increasing sensitivity to certain types of both negative and positive social experience.

\section{Effects of Inflammation on Social Behavior in Humans}

Although animal work provides us with clues regarding how inflammation alters social behavior, only work with humans can reveal how inflammation alters social experience. Thus, while it has been commonly accepted that inflammation induces social withdrawal, it has not been clear what the experiential correlates of this state are, or what cognitive or motivational factors motivate an individual to engage in social withdrawal. In other words, what are the experiential correlates of social withdrawal-do individuals feel lonely or just fatigued? Moreover, what are the cognitive or motivational factors that lead to social withdrawal-a greater sensitivity to negative social experience? A reduced sensitivity to positive social experience? Recent work examining both self-reported experience following an inflammatory challenge as well as neural measures of sensitivity to various kinds of social tasks has helped to uncover the more specific ways in which inflammation can shape social behavior in humans.
The first study that focused specifically on the effect of inflammation on social experience exposed subjects to an experimental inflammatory challenge (ie, endotoxin) and then asked subjects to report on their feelings at hourly intervals (Eisenberger et al, 2009, 2010b). In addition to being asked to report on changes in depressed mood, subjects were also asked to report on their feelings of social disconnection (eg, 'I feel disconnected,' 'I feel like being alone,' and 'I feel overly sensitive around others'). Results from this work showed that the experimental inflammatory challenge, in addition to inducing depressed mood (which had been shown previously) (Reichenberg et al, 2001), also increased feelings of social disconnection (Eisenberger et al, 2010b). A similar study, using a more comprehensive assessment of social disconnection that also included feelings of loneliness (eg, 'I feel lonely,' and 'I feel isolated from others') replicated this finding and showed that the effect of inflammation on social disconnection was stronger for females than males (Moieni et al, 2015c). Moreover, these effects remain after controlling for self-reported sickness symptoms (Eisenberger et al, 2010b; Moieni et al, 2015c), suggesting that the self-reported decrements in felt social disconnection are not simply due to subjects feeling more sick. Similar findings have demonstrated that an inflammatory challenge can increase self-reported social anhedonia ('I want to be alone'; Hannestad et al, 2011). Together, this work suggests that feelings of social disconnection and loneliness (rather than something like simple fatigue) may be the experiential correlate of social withdrawal.

In addition to investigating how inflammation alters self-reported social experience, we can also examine how inflammation alters neural sensitivity to different kinds of social experiences. To date, two broad categories of social experience have been found to be altered as a function of inflammation. Specifically, inflammation alters neural sensitivity to negative social stimuli or experiences (eg, social rejection, receiving negative social feedback, and viewing threatening faces; Eisenberger et al, 2009; Inagaki et al, 2012; Muscatell et al, 2016) as well as sensitivity to positive social stimuli or experiences (eg, viewing close others and receiving positive social feedback; Inagaki et al, 2015; Muscatell et al, 2016). A heightened sensitivity to both positive and negative social stimuli may help an individual to more quickly determine which individuals might be supportive and provide help in times of need and which individuals will not and should be avoided.

\section{Neural Sensitivity to Negative Social Experience}

Studies of how inflammatory activity alters sensitivity to negative social experience have consistently shown that inflammation heightens neural sensitivity to several different types of negative social stimuli. In one study, subjects were randomly assigned to either receive an experimental inflammatory challenge (endotoxin) or not, and then completed a social exclusion task in which they were socially excluded during a virtual ball-tossing game (Cyberball; 

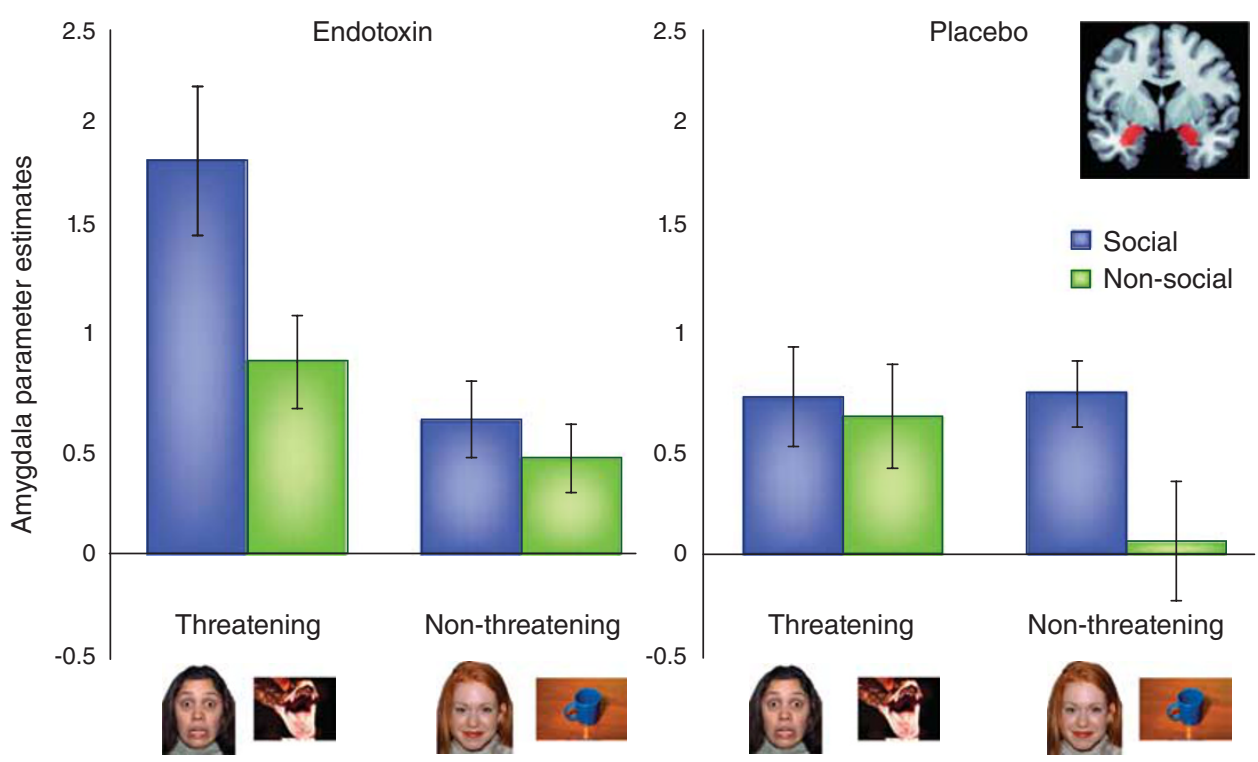

Figure 2. Endotoxin increases amygdala activity specifically to socially threatening images. This figure comes from a prior study (Inagaki et al, 2012) and shows amygdala activity (average of left and right sides) in response to viewing social and non-social, threatening and non-threatening images, displayed separately for endotoxin and placebo subjects.

Eisenberger et al, 2003). Results demonstrated that subjects who showed a greater increase in proinflammatory cytokines in response to the inflammatory challenge also showed greater activity in neural regions associated with the distressing experience of physical pain (Rainville et al, 1997), namely the dorsal anterior cingulate cortex (dACC) and anterior insula (Eisenberger et al, 2009). Hence, greater inflammatory activity to an inflammatory challenge was associated with greater neural sensitivity to social exclusion.

Using a similar inflammatory challenge paradigm, another study investigated how endotoxin alters neural responses to negative social feedback from an evaluator. Results from this study revealed that, in response to negative ( $v s$ neutral) feedback, subjects exposed to the inflammatory challenge showed greater activity in the dACC as well as the amygdala, a neural region that responds, in part, to threat (Muscatell et al, 2016). Thus, again, an inflammatory challenge led to increased sensitivity to a negative social experience.

Finally, other work has examined how an inflammatory challenge affects neural sensitivity specifically to threatening social stimuli (eg, images of fearful faces), compared with threatening non-social stimuli (eg, images of snakes) as well as matched control stimuli (non-threatening social images: happy faces, and non-threatening non-social images: household objects; Inagaki et al, 2012). Based on work showing that both social and non-social threatening stimuli activate the amygdala (Hariri et al, 2002), analyses examined whether endotoxin, as compared with placebo, led to greater activation of the amygdala in response to these socially threatening images. Interestingly, although there were no differences between the endotoxin and placebo groups in amygdala responses to the non-social, threatening images, results showed a differential effect on responses to the socially threatening images. Specifically, exposure to endotoxin induced increased amygdala activity to the socially threatening images relative to all the other trial types (Figure 2). Moreover, greater endotoxin-induced amygdala activity in response to social $v s$ non-social threatening images was associated with greater increase in feelings of social disconnection. Thus, endotoxin led to a specific increase in sensitivity to threatening social (but not threatening, non-social) stimuli, suggesting that inflammation may specifically enhance sensitivity to the danger of the social world, possibly for self-protective purposes. It has also been suggested that inflammation-induced sensitivity to socially threatening stimuli may be one mechanism that helps to promote social withdrawal from unfriendly others during times of sickness (Inagaki et al, 2012), which may not only be self-protective, but may also help to curtail the spread of illness or infection through social networks (Cole, 2006).

\section{Neural Sensitivity to Positive Social Experience}

In addition to examining how inflammation alters neural sensitivity to negative social stimuli and experiences, recent research has also started to examine how inflammation affects neural sensitivity to positive social stimuli and experiences as well. Given that inflammation is known to induce social withdrawal, one might assume that this might be mediated in part by a reduction in reward-related neural sensitivity to positive social experience. In other words, inflammation might reduce reward-related reactivity to positive social events, leading to a reduced interest in the social world and ultimately to social withdrawal. Indeed, this hypothesis fits with other research findings showing that inflammation: (1) induces depressive symptoms, which are 

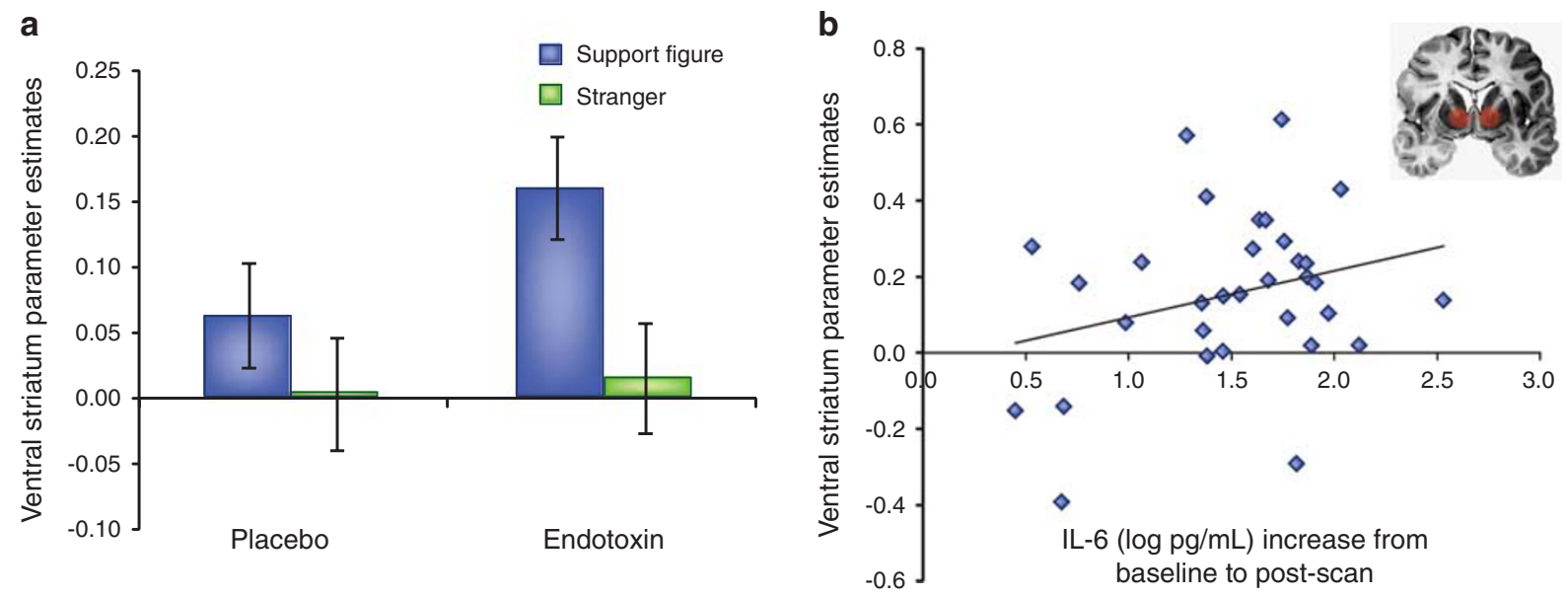

Figure 3. Endotoxin increases reward-related neural activity in response to viewing images of close others. These data come from a prior study (Inagaki et al, 2015) showing that: (a) endotoxin (vs placebo) led to increased activity in the ventral striatum (VS) in response to viewing images of social support figures and (b) greater increases in IL-6 responses to the endotoxin challenge were associated with greater increases in VS activity to viewing social support figures.

characterized by anhedonia or an inability to experience reward and (2) leads to a reduction in reward-related neural reactivity to monetary rewards (Eisenberger et al, 2010a). Although it seems logical to assume that inflammation would reduce reward-related neural reactivity to various types of positive experiences, a different picture emerges when we focus on positive social experiences. Here, consistent with the finding that animals actually spend more, rather than less, time with close others when sick (Hennessy et al, 2014), mounting evidence in humans shows that inflammation actually seems to lead to increased approach-related behavior and reward-related neural activity in response to close others. These findings are in line with the idea that approaching close others during times of sickness, in order to obtain additional help and care, may be more adaptive for survival than withdrawing from them during this vulnerable time.

In one study, subjects exposed to endotoxin ( $v s$ placebo) reported a greater desire to be with their close others during the time of the peak inflammatory response. Moreover, subjects exposed to endotoxin ( $v s$ placebo) showed greater neural activity in the ventral striatum (VS), a key rewardrelated neural region, in response to viewing images of their loved ones (there were no differences in viewing images of strangers; Inagaki et al, 2015). Finally, those who showed greater increases in circulating cytokines (interleukin-6; IL-6) in response to endotoxin also showed greater activity in the VS in response to viewing images of loved ones (Figure 3). In sum, those exposed to inflammation showed increased reward-related neural activity in response to close others.

Similarly, another study investigated how inflammation altered reward-related neural activity, this time in response to hearing positive feedback from an evaluator (Muscatell et al, 2016). Although the person providing the feedback was not a close other or social support figure, results showed that subjects exposed to endotoxin ( $v s$ placebo) displayed more reward-related activity in the VS as well as in the ventromedial prefrontal cortex, another reward-related neural region, in response to receiving positive feedback (compared with neutral feedback). Thus, here, inflammation increased reward-related neural reactivity to positive social feedback, which may indicate that inflammatory processes heighten an individual's sensitivity to detecting who might be an 'ally' and thus potentially able to provide support or help during times of sickness and vulnerability.

Together, these findings shed new light on the effects of inflammation on social behavior. In line with some of the research in animals, the findings emphasize the fact that inflammation does not unilaterally induce social withdrawal. Instead, in some cases, sickness may induce a drive to approach close others in order to elicit care and help during times of need. Interestingly, this is consistent with attachment theory, which posits that during times of sickness or need, a child will actually become more sensitive to the whereabouts of the caregiver and will spend more time in close proximity to the caregiver in order to receive more care and support during these times (Bowlby, 1969). The findings here also indicate that, in addition to enhancing approachrelated motivation to close others, sickness may also make individuals more sensitive to who is likely to provide support during times of need (ie, those who provide positive feedback).

\section{Implications of Studying the Effects of Inflammation on Social Behavior}

Given that humans are a highly social species who depend on others for survival, particularly during times of sickness, it is somewhat surprising that most models of inflammation and sickness behavior have largely overlooked the social behavioral consequences of inflammation. Here, we reviewed evidence to show that individuals in a sick state may become particularly sensitive to the social environment. Specifically, 


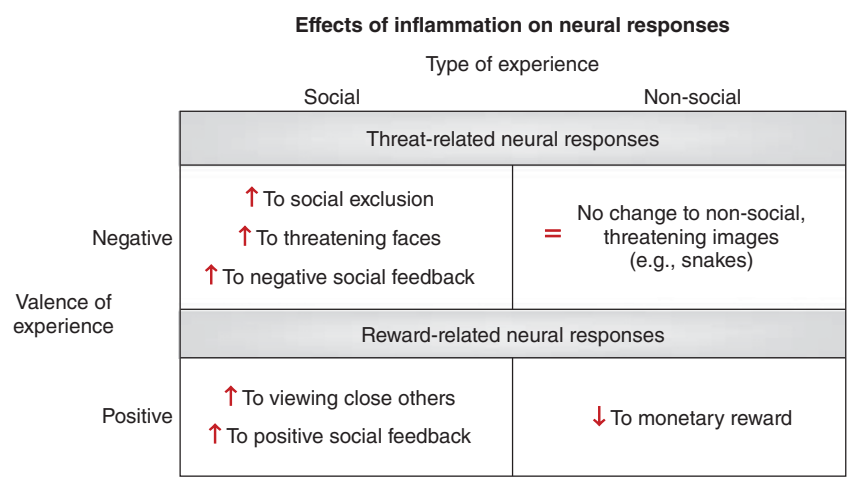

Figure 4. Figure summarizing the effects of experimental inflammatory activity on neural responses, depending on the type of experience (social vs non-social) and the valence of the experience (negative vs positive).

those exposed to an inflammatory challenge showed: (1) enhanced threat-related neural sensitivity to negative social stimuli, but not to non-social, negative stimuli and (2) enhanced reward-related neural sensitivity to positive social stimuli, but reduced reward-related responding to nonsocial, positive stimuli (eg, money; Figure 4).

Importantly, a better and more nuanced understanding of the role that inflammation plays in modifying social behavior may help us to better understand some surprising relationships between inflammation and certain psychiatric disorders that involve altered social sensitivity or compromised social processing. For instance, emerging research has shown that inflammatory processes contribute to certain psychiatric disorders such as depression. Understanding the social impact of inflammation may help us to better understand why inflammation would contribute to these disorders.

\section{Inflammation and Depression}

Mounting evidence has demonstrated a relationship between inflammation and depression (Miller et al, 2009a; Raison et al, 2006; Schiepers et al, 2005). Initial support for this relationship came from observations that $50 \%$ of cancer patients developed depression after undergoing treatments that increased proinflammatory activity (eg, interferonalpha; Bonaccorso et al, 2000; Capuron and Miller, 2004; Raison et al, 2005). Subsequent correlational studies have shown that depressed individuals have elevated levels of proinflammatory cytokines compared with healthy controls (Dowlati et al, 2010; Howren et al, 2009). Finally, experimental inflammatory challenges (endotoxin) also lead to increases in depressed mood in otherwise healthy subjects (Reichenberg et al, 2001; Eisenberger et al, 2009). However, how inflammation leads to a full-blown depressive episode is not yet fully understood.

Although most individuals (80\%) exposed to cytokinebased treatments develop certain types of sickness behavior, such as fatigue, only $21-58 \%$ go on to develop depression (Raison et al, 2005). Thus, other vulnerability factors may play a role in the link between inflammation and depression. Given the important role that feelings of social disconnection (eg, loneliness) play in contributing to depression (Heinrich and Gullone, 2006), it is possible that the social consequences of inflammation may be a critical missing piece in understanding the link between inflammation and depression. Indeed, work has shown that increased feelings of social disconnection in response to an inflammatory challenge mediate the relationship between exposure to the inflammatory challenge and increases in depressed mood (Eisenberger et al, 2010b). Moreover, females, who tend to have higher rates of depression, also show larger increases in feelings of social disconnection in response to an inflammatory challenge than males (Moieni et al, 2015c). Hence, the social consequences of inflammation (ie, increased feelings of social disconnection) may play an important role in understanding the link between inflammation and depression. Additional research on this topic is needed to better understand if those more sensitive to the social consequences of inflammation are more likely to become clinically depressed.

\section{Differentiating Inflammatory-Mediated Depression from Other Forms of Depression}

Although research has clearly shown a relationship between inflammation and depression, more recent accounts have stipulated that not all forms of depression are inflammatorymediated and that instead there may be an inflammatory subtype' of depression that can be distinguished from a noninflammatory subtype (Raison and Miller, 2011). However, distinguishing this 'inflammatory subtype' of depression from other non-inflammatory subtypes has been more challenging, and currently there are no behavioral or neural phenotypic data for understanding the basis of these differences, which is necessary for precision-based intervention development (although C-reactive protein (CRP) levels have been used as a physiological biomarker for this difference; Raison et al, 2013).

Based on newer work examining the social consequences of inflammation, it is possible that neural sensitivity to certain social targets may provide a useful biomarker for discriminating between inflammatory $v s$ non-inflammatory forms of depression. For instance, although major depression is often characterized by social withdrawal and a lack of interest in the social world, inflammatory-induced depression may be different. For instance, work has shown that exposure to an experimental inflammatory challenge ( $v s$ placebo), which increases depressed mood, also leads to: (1) an increased desire to be around loved ones and 2) increased rewardrelated neural activity to viewing images of close others (Inagaki et al, 2015). It is possible, then, that this increase in reward-related neural activity to close others is specific to the 'inflammatory subtype' of depression given the evolutionary benefit of approaching close others during times of sickness.

However, to date, no research has examined how depressed individuals respond neurally to images of close others, but instead has focused on neural responses to monetary reward, happy faces of strangers, or positive words (Zhang et al, 2013). Future work will be needed to examine 
whether reward-related neural activity to close others differentiates between these two forms of depression, with increased VS activity to close others in the inflammatory subtype and reduced VS activity in the non-inflammatory subtype. Understanding the potentially different mechanisms underlying inflammatory- and non-inflammatory forms of depression is critical not only for distinguishing between them, but also for determining appropriate types of treatment. To the extent that sensitivity to certain types of social rewards (eg, close others) is preserved in inflammatory-related depression, this hypothesis suggests a spared island of motivational significance for these individuals, which paves the way for new treatments that better incorporate support or care from close others for those with the inflammatory subtype of depression.

\section{PART II: SOCIAL BEHAVIOR AS AN ORGANIZER OF INFLAMMATORY ACTIVITY}

Just as inflammatory activity can alter social behavior, so too can social behavior and features of the social environment alter inflammatory activity. Specifically, research from animal and human subjects has shown that various types of social stressors, such as those involving social separation, social defeat, social rejection, social loss, or social evaluation, can lead to transient or sometimes longer-lasting increases in proinflammatory activation. This is not to say that other stressors (ie, painful stimulation) do not activate proinflammatory responses, but rather that social stressors are another category of stimuli that can.

To understand why social factors can increase inflammatory activity, it is important to know that inflammation is regulated at multiple levels. First, inflammation is regulated by factors in the periphery, such as exposure to an extracellular pathogen, which then leads to the activation of inflammatory-related transcription factors and the increased production of proinflammatory cytokines (Irwin and Cole, 2011). However, inflammation is also regulated neurally, which allows inflammatory processes to be shaped by certain features of the environment that predict a greater likelihood of wounding and infection and thus a greater need for inflammatory activity. These threatening features are processed neurally, resulting in sympathetic nervous system (SNS) activation as well as activation of the hypothalamicpituitary-adrenocortical (HPA) axis, which can then influence inflammatory responding (Irwin and Cole, 2011; Eisenberger and Cole, 2012). (Specifically, under normal conditions, acute SNS activation enhances inflammatory activity, whereas acute HPA activation inhibits inflammatory responding (Eisenberger and Cole, 2012). However, under conditions of chronic stress, the repeated activation of these systems can bias an individual to increased inflammatory activity. Specifically, whereas SNS nerve fibers that innervate lymph nodes and coordinate immune responses are upregulated in response to chronic stress (Sloan et al, 2007), the HPA axis and its glucocorticoid outputs can become less effective in reducing proinflammatory activity, a process referred to as glucocorticoid resistance (Avitsur et al, 2001), and can thus lead to greater proinflammatory activity). Given the importance of social ties for mammalian (and particularly primate) survival, threats to social connection-such as experiences of rejection, isolation, conflict, or loss-signal that one is more vulnerable and may face a greater likelihood of wounding and infection and thus a greater need for inflammatory activity. Indeed, all of these factors have been associated with increased inflammatory activity.

Recent models have suggested that, through neuralimmune connections, the immune system may have evolved to 'listen in' to certain features of the social environment to anticipate what kinds of immune processes might be most needed and to redirect energy and resources to those critical needs (Cole, 2013, 2014; Eisenberger and Cole, 2012). To the extent that a lack of social connection is a survival risk, leading individuals to be more vulnerable to attack (by predators and hostile conspecifics) and thus more prone to wounding and infection, the body may respond to these social stressors by upregulating proinflammatory activity. At the same time, a lack of social connection may also signify a reduced risk of viral infections, since these infections are transmitted through social contact. Hence, the body may respond to these same social stressors by down-regulating antiviral responses, which are less needed. This pattern of social stress-induced up-regulation of inflammatory activity and down-regulation of antiviral responses has been termed the conserved transcriptional response to adversity (CTRA) (Cole, 2013, 2014) and is conceptualized as an evolutionary adaptive response to the threat of social disconnection. Although adaptive on some level, activation of the CTRA can also increase an individual's risk for inflammatory-mediated diseases as well as viral infections.

A more detailed account of the specific mechanisms whereby social factors regulate immune system responses is presented elsewhere (Irwin and Cole, 2011; Slavich and Cole, 2013; Slavich and Irwin, 2014). Here, we summarize research that highlights several of the consequences of having an immune system that is sensitive to features of the social environment. Specifically, we review work showing: (1) that exposure to real-world or lab-based social stressors (eg, parental separation, rejection, negative interactions and social evaluative stress) increases proinflammatory activity, (2) that individuals who are either objectively socially isolated or subjectively socially isolated (ie, lonely) show increased proinflammatory activity, and (3) that individuals who tend to be more socially disconnected show increased proinflammatory activity to an inflammatory challenge or social stressor. (Although not the focus of this review, additional evidence highlighting the sensitivity of the immune system to features of the social environment comes from studies showing that the mere perception of diseaserelated cues in others (eg, sick individuals) can lead to increases in inflammatory responses in the observer (Schaller et al, 2010; Stevenson et al, 2012).) 


\section{Exposure to Social Stress Increases Inflammatory Activity}

To the extent that mammals have evolved to have an immune system that is sensitive to the social world, there are a few consequences that should be observed. One of the first and most basic consequences is that various types of social stressors should prime the immune system to increase proinflammatory activity to prepare for these more vulnerable situations. Indeed, both studies of exposure to realworld social stressors as well as laboratory-based social stressors have provided evidence for this first consequence.

\section{Exposure to Real-World Social Stress}

Just within the past several years, significant strides have been made to show that real-world social stressors, events such as parental separation in early life or bereavement in late life, lead to increases in proinflammatory activity. Indeed, the responsiveness of inflammatory activity to these social stressors has now been demonstrated across the lifespan.

For instance, children younger than 8 years old who experienced more stressful life events that were social in nature (including parental separation, being taken into foster care, or physical/sexual abuse) showed elevated markers of inflammation (CRP) both 2 and 7 years later (Slopen et al, 2013). In adolescence, a similar picture emerges. Adolescents who reported having more negative social interactions with friends and family members showed higher levels of inflammatory activity (CRP; Fuligni et al, 2009; greater LPSstimulated production of IL-6; Miller et al, 2009b). Moreover, female adolescents who reported experiencing episodes of targeted rejection evidenced higher levels of proinflamatory gene expression than those who did not (Murphy et al, 2013). Similarly, college students who reported experiencing more negative and competitive social interactions on a daily basis showed higher levels of IL- 6 and of the soluble receptor for tumor necrosis factor- $\alpha$ (sTNF $\alpha$ RII; Chiang et al, 2012). Not surprisingly, these effects are observed in response to later life stressors as well. Thus, older adults who recently lost a spouse had levels of inflammatory activity (interleukin-1 receptor antagonist (IL-1Ra), IL-6) that were 1.5 times higher than older adults who had not lost a spouse (Schultze-Florey et al, 2012), and this effect was moderated by the presence of a cytokine gene polymorphism (ie, IL-6 -174 SNP). In other words, genetic variability interacts with an environmental stressor, leading to increased inflammatory markers in genetically susceptible individuals. Altogether, these findings support the idea that real-world social stressors across the lifespan can potentially signal the immune system to increase proinflammatory activity of various kinds.

\section{Exposure to Laboratory-Based Social Stress}

Laboratory studies provide a nice complement to real-world studies of stress-induced inflammatory activity by allowing us to examine the causal influence of social stressors on inflammatory responses. Overall, these studies have shown that various types of social stressors can reliably elicit inflammatory activity in a laboratory setting (for a review and meta-analysis, see Steptoe et al, 2007) and that greater experiences of social stress in response to these stressors are associated with greater increases in inflammatory responding.

For instance, one of the first studies to examine the inflammatory consequences of social stress had individuals write about an experience that induced feelings of shame, an emotion signifying that one is bad or unlovable (Dickerson et al, 2004). Findings showed that subjects who wrote about a shameful experience $v s$ those who wrote about a control topic showed larger increases in inflammatory activity, as indexed by greater increases in sTNF $\alpha$ RII. Moreover, those who reported the greatest increases in shame in response to the task showed the largest increases in sTNF $\alpha$ RII.

Other studies have examined the effect of more standardized social stressors on inflammatory responding. In one of these studies, subjects completed the Trier Social Stress Test (TSST), which involves giving an impromptu speech and doing mental arithmetic in front of an evaluative panel. In this study, however, subjects either completed the TSST in the standard way-in front of an evaluative panel-or they completed it alone, which eliminates the social-evaluative stress (Dickerson et al, 2009). Results showed that, compared with subjects who completed the TSST alone, those who completed the TSST in front of an evaluative panel showed larger increases in proinflammatory activity from pre to post-stress (ie, an increase in stimulated production of TNF- $\alpha$ ). Moreover, greater perceptions of being evaluated were directly associated with greater increases in inflammatory activity.

Indeed, several other studies have shown that the TSST can increase proinflammatory responses from pre- to post-stress (Moons et al, 2010; Slavich et al, 2010: Yamakwa et al, 2009) and that greater experiences of social stress are associated with greater increases in inflammatory responding. For instance, those who reported higher levels of fear or anxiety in response to the TSST also showed greater increases in inflammatory responses to the TSST (IL- 6 and sTNF $\alpha$-RII: Moons et al, 2010; IL-6: Carroll et al, 2011). Similarly, those who reported a greater perceived intensity of stress also showed larger increases in inflammatory activity to the TSST (IL-1 $\beta$; Yamakawa et al, 2009). Finally, in a neuroimaging study, individuals who showed more social pain-related neural activity in response to social exclusion (dACC, anterior insula) also showed larger increases in sTNF $\alpha$-RII in response to the TSST (Slavich et al, 2010), suggesting that those who are most sensitive to social rejection may also be the most likely to show increased inflammatory activity in response to the possibility of social rejection (in the TSST).

\section{Social Isolation is Associated with Increased Inflammatory Activity}

A second possible consequence of having an immune system that is sensitive to the social world is that individuals who tend 
to feel more socially disconnected should show greater baseline inflammatory activity. Indeed, studies of older adults have shown that those who are objectively more socially isolated or subjectively more socially isolated (eg, lonely) tend to have increased levels of proinflammatory activity.

In one study, older men with the fewest social ties were approximately two times more likely to have elevated CRP levels than men with the most social ties (although this effect was not observed in older women) (Ford, Loucks, and Berkman, 2006). Similarly, in an adult sample of both males and females (over 40 years old), those who were the most socially isolated were 2.5 times more likely to have elevated CRP levels compared with those individuals who were the most socially integrated (Heffner et al, 2011). Comparable effects have been observed in other studies of older adults as well (Shankar et al, 2011). Finally, in addition to objective social isolation, subjective social isolation or the experience of being lonely has also been shown to be associated with increased inflammation. In a landmark study, Cole and colleagues demonstrated that older individuals with high levels of loneliness showed the classic CTRA profile, namely, an increase in proinflammatory gene expression along with a decrease in antiviral gene expression (Cole et al, 2007).

Thus, across multiple studies, there appears to be a reliable relationship between either objective or subjective social isolation and increased inflammation. Interestingly, these findings typically emerge in older adult samples and have not been observed in the same manner in younger samples (Moieni et al, 2015a). Hence, for younger individuals, it is possible that social isolation or loneliness only triggers an increase in inflammatory activity in response to another stressor but is not associated with higher baseline levels of inflammation. Indeed, several studies in both animal and human samples have shown that individuals who tend to be sensitive to social disconnection or social stress show greater increases in inflammation when faced with either an inflammatory challenge or another social stressor (reviewed below).

\section{Social Disconnection Predicts Greater Inflammatory Responding to Stress}

The final consequence of a socially sensitive immune system is that to the extent that an individual tends to be socially isolated (subjectively and objectively), that individual may need to cultivate a larger inflammatory response to deal with various stressors. The idea here is that a socially isolated individual may either have less care or help, or may be more vulnerable to attack and thus may need to cultivate a greater inflammatory response to deal with stressors.

Along these lines, both human and animal research demonstrates that those who experience more social stress, or who are more sensitive to various forms of social threat, also show larger inflammatory responses to an endotoxin challenge. For instance, Moieni et al (2015a) have shown that individuals who tend to be more sensitive to social disconnection (a composite measure of loneliness, anxious
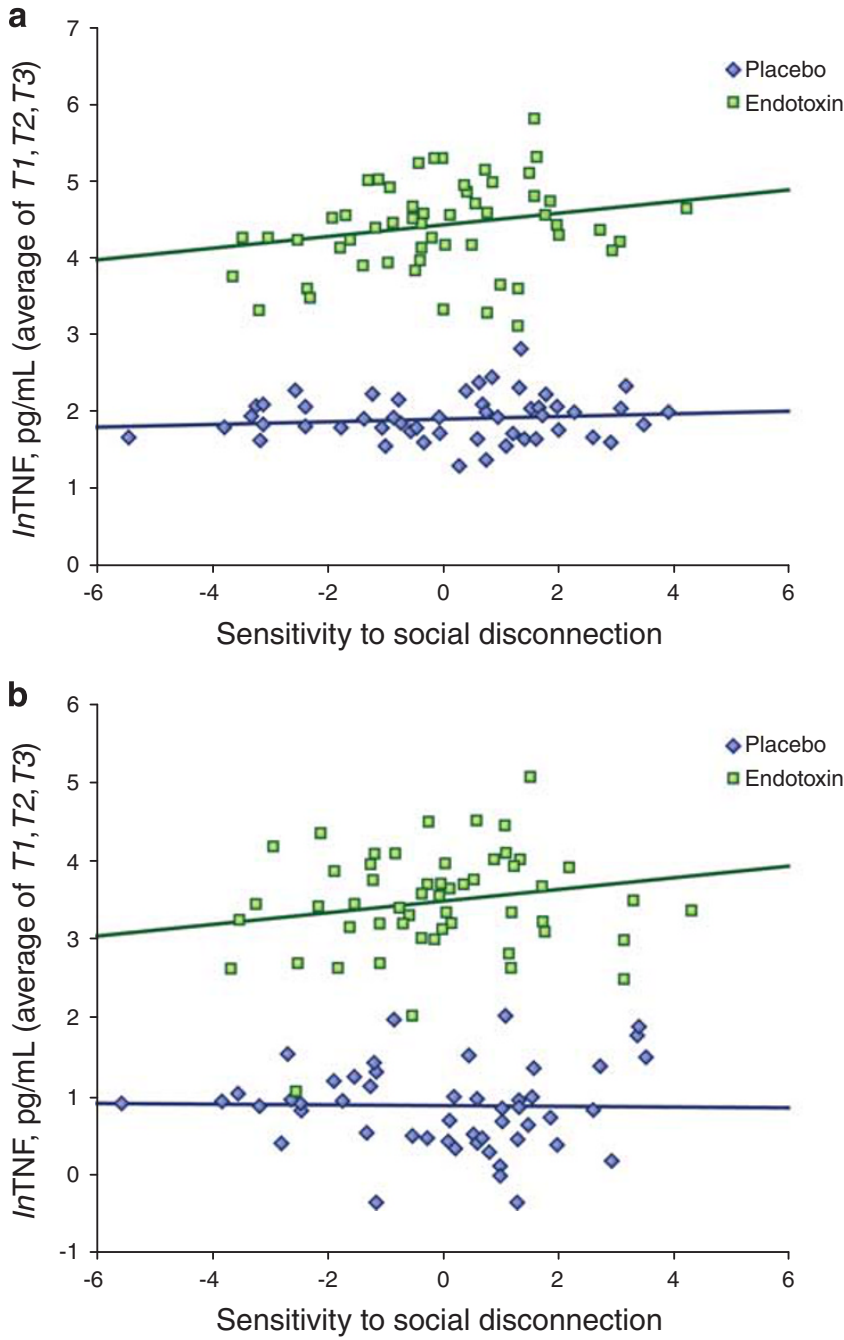

Figure 5. Trait sensitivity to social disconnection is associated with greater increases in inflammatory activity to endotoxin, but not placebo. This figure is reprinted from a prior study (Moieni et al, 2015a), which showed that individuals who reported higher levels of sensitivity to social disconnection showed greater increases in (a) TNF and (b) IL-6 in response to endotoxin (TNF: $\mathrm{B}=0.077, t=2.84, p<0.01$; $I L-6: \mathrm{B}=0.074$, $t=2.02, p<0.05$ ) but not in response to placebo (TNF: $\mathrm{B}=0.021$, $t=0.868, p=0.39 ; / L-6: \mathrm{B}=-0.005, t=-0.139, p=0.89$ ).

attachment, fear of negative evaluative, and rejection sensitivity) show larger increases in circulating proinflammatoy cytokines (Figure 5), as well as greater increases in proinflammatory gene expression, in response to a low-dose endotoxin challenge.

Similar results have been observed in animals; however, these studies have demonstrated that it is only social stress, but not non-social stress, that increases inflammatory responses to an endotoxin challenge. Thus, in one study, social stress (chronic social defeat), but not physical stress (restraint stress), more than doubled the mortality rate of mice exposed to a septic dose of LPS (Quan et al, 2001). Moreover, this increased mortality was associated with increased inflammatory histopathology in the brain, lung, liver, and spleen as well as increased glucocorticoid resistance (ie, reduced sensitivity of immune cells to the 
anti-inflammatory effects of glucocorticoids) in the splenocytes. Similarly, rats exposed to repeated social defeat stress who received endotoxin showed greater increases in IL-1 $\beta$ and increased rates of mortality compared with unstressed rats who received endotoxin (Carobrez et al, 2002). Finally, mice subjected to a different social stressor-prolonged social isolation followed by social regrouping-but not mice subjected to physical stressors (restraint, tail pinch, loud noise), showed enhanced IL-6 and $\mathrm{TNF} \alpha$ responses to endotoxin (Gibb et al, 2008).

In addition to impacting responses to an inflammatory stressor, higher baseline levels of social sensitivity or social stress may also impact inflammatory responses to a social stressor. Although research on this topic is more sparse, studies have shown that older women higher in loneliness showed greater increases in IL-6 and IL-1Ra in response to a mental stressor (Hackett et al, 2012). Moreover, adults higher in loneliness showed increased stimulated cytokine production in response to a stress task (Jaremka et al, 2013). Together, these studies show that trait social sensitivity or higher baseline levels of social stress may have an important impact on inflammatory responses to both inflammatory and social stressors.

\section{Implications of Studying the Effects of Social Behavior on Inflammation}

Though somewhat surprising, research on the effects of social behavior on inflammation suggests that how we engage with the social world has implications for the landscape of our immune system. Specifically, when an individual is disconnected from the social group, through acute events such as rejection or evaluation, or through longterm situational variables such as social isolation or loneliness, there may be a priming of the immune system to increase inflammatory activity and to prepare for these more vulnerable situations when an individual is alone. Along these lines, we have reviewed evidence here for three consequences of this socially-sensitive immune system, namely: (1) that social stressors can increase inflammation, (2) that socially isolated individuals tend to have increased levels of inflammation, and (3) that those who chronically face more isolation or social stress show greater inflammatory responses to other acute stressors.

Understanding the impact of the social environment on the immune system highlights a few important considerations. First, these findings suggest that isolated or lonely individuals, particularly older adults, may be at a greater risk for various inflammatory-related diseases (eg, arthritis, diabetes, obesity, atherosclerosis, and certain cancers). Because of this, it may be important for doctors to assess an individual's level of objective or subjective social isolation, in addition to assessing routine risk factors for health (eg, blood pressure and sedentary lifestyle). Not only could an assessment of social isolation be an important predictor of disease, but identifying socially isolated individuals could also prompt attempts at interventions for promoting social ties (with the hope of curtailing risk for some inflammatoryassociated diseases).

Another implication of these findings is that they suggest that certain individuals are more likely to be negatively impacted by certain proinflammatory stressors or social stressors. Thus, individuals who are higher in feelings of social disconnection or loneliness may be the ones who show larger inflammatory responses and ultimately get more sick in response to infections. They may also be more vulnerable to inflammatory responses to social stressors. This suggests that socially disconnected or lonely individuals may have a more severe course of illness in response to infections, a more severe inflammatory response to social stressors, and may ultimately have a greater risk of depressive symptoms in response to this exaggerated inflammatory response (Aschbacher et al, 2012). Hence, the combination of being low in social connection combined with exposure to either an inflammatory challenge or a potent social stressor may increase risk for depression through inflammatory mechanisms. Although previous studies have highlighted the depressogenic effects of exposing vulnerable individuals to social stress (Monroe and Simons, 1991), less research has examined the depressogenic effects of exposing vulnerable individuals to inflammatory stress. This might be an important avenue for future research.

\section{CONCLUSIONS}

Although inflammation and social behavior appear to be unlikely bedfellows, they are intricately connected. Here, we have reviewed considerable evidence: (1) that the immune system is a powerful regulator of social behavior and (2) that social behavior or features of the social environment are powerful regulators of the immune system. Indeed, this coregulation of social behavior and immune system activity, particularly inflammatory responses, makes good sense.

When we are sick, we need to be especially sensitive to the social world in order to more carefully navigate our interactions with potentially dangerous strangers and increase our connection to those who we know will help us during this time of need. Going forward, it will be important for research in this area to more carefully delineate the effects of sickness on different types of social behaviors as there may be different effects depending on whether the social target is a stranger or a close other as well as whether the social interaction is positive or negative. To date, these differences have not been carefully examined leading to the incorrect conclusion that sickness uniformly elicits social withdrawal.

Likewise, given the powerful role that social relationships play in our survival and the fact that social isolation makes us more vulnerable to attack and wounding, the immune system may have evolved to prepare for threats to social connection by increasing efforts towards heightened proinflammatory responses in these more vulnerable situations. Future work will be necessary to: (1) identify the types of social stressors that are the most potent activators of the 
immune system as well as (2) investigate whether there are specialized mechanisms whereby social stressors influence the immune system or whether these are the same mechanisms that allow other non-social stressors to influence the immune system as well.

Viewing sickness as a social phenomenon, in addition to a physical phenomenon, may help us to better understand emerging relationships between inflammation and mental health problems like depression (Miller et al, 2009a). Likewise, viewing social stress or social isolation as a physiological phenomenon, as well as a psychological phenomenon, may help us to better understand the robust relationships between social ties and health (Holt-Lunstad et al, 2010). A better understanding of the co-regulation of inflammation and social behavior may bring us a step closer to these goals.

\section{FUNDING AND DISCLOSURE}

This research was funded, in part, by an R01 from NIMH to NIE (5R01MH091352). The authors declare no conflict of interest.

\section{ACKNOWLEDGMENTS}

We acknowledge the additional support provided by a predoctoral NRSA individual fellowship from NIA (1F31AG048668) to $\mathrm{MM}$ as well as the support provided by R01AG034588; R01AG026364; R01CA160245-01; R01CA119159; R01HL095799; R01DA032922; and the Cousins Center for Psychoneuroimmunology to MRI.

\section{REFERENCES}

Aschbacher K, Epel E, Wolkowitz OM, Prather AA, Puterman E, Dhabhar FS (2012). Maintenance of a positive outlook during acute stress protects against proinflammatory reactivity and future depressive symptoms. Brain Behav Immun 26: 346-352.

Avitsur R, Stark JL, Sheridan JF (2001). Social stress induces glucocorticoid resistance in subordinate animals. Horm Behav 39: 247-257.

Bilbo SD, Klein SL, DeVries AC, Nelson RJ (1999). Lipopolysaccharide facilitates partner preference behaviors in female prairie voles. Physiol Behav 68: 151-156.

Bluthé RM, Dantzer R, Kelley KW (1992). Effects of interleukin-1 receptor antagonist on the behavioral effects of lipopolysaccharide in rat. Brain Res 573: 318-320.

Bluthé RM, Pawlowski M, Suarez S, Parnet P, Pittman Q, Kelley KW et al (1994). Synergy between tumor necrosis factor $\alpha$ and interleukin-1 in the induction of sickness behavior in mice. Psychoneuroendocrinology 19: 197-207.

Bonaccorso S, Metzer H, Maes M (2000). Psychological and behavioral effects of interferons. Curr Opin Psychiatry 13: 673-677.

Bowlby J (1969). Attachment. Attachment and Loss Vol. 1. Basic Books: New, York, NY.

Capuron L, Miller AH (2004). Cytokines and psychopathology: lessons from Interferon-alpha. Biol Psychiatry 56: 819-824.

Carobrez SG, Gasparotto OC, Buwalda B, Bohus B (2002). Long-term consequences of social stress on corticosterone and IL-1 $\beta$ levels in endotoxinchallenged rats. Physiol Behav 76: 99-105.

Carroll JE, Low CA, Prather AA, Cohen S, Fury JM, Ross DC et al (2011). Negative affective responses to a speech task predict changes in interleukin (IL)-6. Brain Behav Immun 25: 232-238.

Chiang JJ, Eisenberger NI, Seeman TE, Taylor SE (2012). Negative and competitive social interactions are related to heightened proinflammatory cytokine activity. Proc Natl Acad Sci 109: 1878-1882.

Cole SW (2006). The complexity of dynamic host networks. In: Complex Systems Science In Biomedicine. Springer: US, 605-629.

Cole SW (2013). Social regulation of human gene expression: mechanisms and implications for public health. Am. J. Public Health 103: S84-S92.
Cole SW (2014). Human social genomics. PLoS Genet 10: e1004601.

Cole SW, Hawkley LC, Arevalo JM, Sung CY, Rose RM, Cacioppo JT (2007). Social regulation of gene expression in human leukocytes. Genome Biol 8: R189.

Dantzer R (2001). Cytokine Induced sickness behavior: Mechanisms and implications. Ann N Y Acad Sci 933: 222-234.

Dantzer R, Kelley KW (2007). Twenty years of research on cytokine-induced sickness behavior. Brain Behav Immun 21: 153-160.

Dantzer R, O'Connor JC, Freund GG, Johnson RW, Kelley KW (2008). From inflammation to sickness and depression: when the immune system subjugates the brain. Nat Rev Neurosci 9: 46-57.

Dickerson SS, Gable SL, Irwin MR, Aziz N, Kemeny ME (2009). Social-evaluative threat and proinflammatory cytokine regulation an experimental laboratory investigation. Psychol Sci 20: 1237-1244.

Dickerson SS, Kemeny ME, Aziz N, Kim KH, Fahey JL (2004). Immunological effects of induced shame and guilt. Psychosom Med 66: 124-131.

Dowlati Y, Herrmann N, Swardfager W, Liu H, Sham L, Reim EK et al (2010). A metaanalysis of cytokines in major depression. Biol Psychiatry 67: 446-457.

Eisenberger NI, Berkman ET, Inagaki TK, Rameson L, Mashal N, Irwin MR (2010a). Inflammation-induced anhedonia: Endotoxin reduces ventral striatum responses to reward. Biol Psychiatry 68: 748-754.

Eisenberger NI, Cole SW (2012). Social neuroscience and health: neurophysiological mechanisms linking social ties with physical health. Nat Neurosci 15: 669-674.

Eisenberger NI, Inagaki TK, Mashal NM, Irwin MR (2010b). Inflammation and social experience: an inflammatory challenge induces feelings of social disconnection in addition to depressed mood. Brain Behav Immun 24: 558-563.

Eisenberger NI, Inagaki TK, Rameson L, Mashal NM, Irwin MR (2009). An fMRI study of cytokine-induced depressed mood and social pain: the role of sex differences. Neuroimage 47: 881-890.

Eisenberger NI, Lieberman MD, Williams KD (2003). Does rejection hurt? An fMRI study of social exclusion. Science 302: 290-292.

Ford ES, Loucks EB, Berkman LF (2006). Social integration and concentrations of C-reactive protein among US adults. Ann Epidemiol 16: 78-84.

Fuligni AJ, Telzer EH, Bower J, Cole SW, Kiang L, Irwin MR (2009). A preliminary study of daily interpersonal stress and C-reactive protein levels among adolescents from Latin American and European backgrounds. Psychosom Med 71: 329-333.

Gibb J, Hayley S, Gandhi R, Poulter MO, Anisman H (2008). Synergistic and additive actions of a psychosocial stressor and endotoxin challenge: circulating and brain cytokines, plasma corticosterone and behavioral changes in mice. Brain Behav Immun 22: 573-589.

Goehler LE, Relton JK, Dripps D, Kiechle R, Tartaglia N, Maier SF et al (1997). Vagal paraganglia bind biotinylated interleukin-1 receptor antagonist: a possible mechanism for immune-to-brain communication. Brain Res Bull 43: 357-364.

Hackett RA, Hamer M, Endrighi R, Brydon L, Steptoe A (2012). Loneliness and stress-related inflammatory and neuroendocrine responses in older men and women. Psychoneuroendocrinology 37: 1801-1809.

Hannestad J, DellaGioia N, Ortiz N, Pittman B, Bhagwagar Z (2011). Citalopram reduces endotoxin-induced fatigue. Brain Behav Immun 25: 256-259.

Hariri AR, Tessitore A, Mattay VS, Fera F, Weinberger DR (2002). The amygdala response to emotional stimuli: a comparison of faces and scenes. Neuroimage 17: 317-323.

Hart BL (1988). Biological basis of the behavior of sick animals. Neurosci Biobehav Rev 12: 123-137.

Heffner KL, Waring ME, Roberts MB, Eaton CB, Gramling R (2011). Social isolation, C-reactive protein, and coronary heart disease mortality among communitydwelling adults. Social Sci Med 72: 1482-1488.

Heinrich LM, Gullone E (2006). The clinical significance of loneliness: a literature review. Clin Psychol Rev 26: 695-718.

Hennessy MB, Deak T, Schiml PA (2014). Sociality and sickness: have cytokines evolved to serve social functions beyond times of pathogen exposure? Brain Behav Immun 37: 15-20.

Holt-Lunstad J, Smith TB, Layton JB (2010). Social relationships and mortality risk: a meta-analytic review. PLoS Med 7: e1000316.

Howren MB, Lamkin DM, Suls J (2009). Associations of depression with C-reactive protein, IL-1, and IL-6: A meta-analysis. Psychosom Med 71: 171-186.

Inagaki TK, Muscatell KA, Irwin MR, Cole S, Eisenberger NI (2012). Inflammation selectively enhances amygdala activity to socially threatening images. Neuroimage 59: 3222-3226.

Inagaki TK, Muscatell KA, Moeini M, Dutcher J, Jevtic I, Irwin MR et al (2015). The role of the ventral striatum in inflammatory-induced approach toward support figures. Brain Behav Immun 44: 247-252.

Irwin MR, Cole SW (2011). Reciprocal regulation of the neural and innate immune systems. Nat Rev Immunol 11: 625-632. 
Jaremka LM, Fagundes CP, Peng J, Bennett JM, Glaser R, Malarkey WB et al (2013). Loneliness promotes inflammation during acute stress. Psychol Sci 24: 1089-1097.

Kelley KW, Bluthe RM, Dantzer R, Zhou JH, Shen WH, Johnson RW et al (2003). Cytokine-induced sickness behavior. Brain Behav Immun 17: S112-S118.

Maier SF, Watkins LR (1998). Cytokines for psychologists: Implications of bidirectional immune-to-brain communication for understanding behavior, mood, and cognition. Psychol Rev 105: 83-107.

Miller AH, Maletic V, Raison CL (2009a). Inflammation and its discontents: the role of cytokines in the pathophysiology of major depression. Biol Psychiatry 65: 732-741.

Miller G, Rohleder N, Cole SW (2009b). Chronic interpersonal stress predicts activation of pro-and anti-inflammatory signaling pathways six months later. Psychosom Med 71: 57-62.

Moieni M, Irwin MR, Jevtic I, Breen EC, Cho HJ, Arevalo JM et al (2015a). Trait sensitivity to social disconnection enhances pro-inflammatory responses to a randomized controlled trial of endotoxin. Psychoneuroendocrinology 62: 336-342.

Moieni M, Irwin MR, Jevtic I, Breen EC, Eisenberger NI (2015b). Inflammation impairs social cognitive processing: a randomized controlled trial of endotoxin. Brain Behav Immun 48: 132-138.

Moieni M, Irwin MR, Jevtic I, Olmstead R, Breen EC, Eisenberger NI (2015c). Sex differences in depressive and socioemotional responses to an inflammatory challenge: implications for sex differences in depression. Neuropsychopharmacology 40: 1709-1716.

Monroe SM, Simons AD (1991). Diathesis-stress theories in the context of life stress research: implications for the depressive disorders. Psychol Bull 110: 406-425.

Moons WG, Eisenberger NI, Taylor SE (2010). Anger and fear responses to stress have different biological profiles. Brain Behav Immun 24: 215-219.

Murphy ML, Slavich GM, Rohleder N, Miller GE (2013). Targeted rejection triggers differential pro-and anti-inflammatory gene expression in adolescents as a function of social status. Clin Psychol Sci 1: 30-40.

Muscatell KA, Moieni M, Inagaki TK, Duthcher JM, Jevtic I, Breen EC et al (2016). Inflammation enhances neural sensitivity to negative and positive social feedback. Brain Behav Immun. (e-pub ahead of print).

Quan N, Avitsur R, Stark JL, He L, Shah M, Caligiuri M et al (2001). Social stress increases the susceptibility to endotoxic shock. J. Neuroimmunol. 115: 36-45.

Quan N, Banks WA (2007). Brain-immune communication pathways. Brain Behav Immun 21: 727-735.

Rainville P, Duncan GH, Price DD, Carrier B, Bushnell MC (1997). Pain affect encoded in human anterior cingulate but not somatosensory cortex. Science 277 : 968-971.

Raison CL, Capuron L, Miller AH (2006). Cytokines sing the blues: inflammation and the pathogenesis of depression. Trends Immunol 27: 24-31.

Raison CL, Demetrashvili M, Capuron L, Miller AH (2005). Neuropsychiatric adverse effects of Interferon-alpha: recognition and management. CNS Drugs 19: 105-123.

Raison CL, Miller AH (2011). Is depression an inflammatory disorder? Curr Psychiatry Rep 13: 467-475.
Raison CL, Rutherford RE, Woolwine BJ, Shuo C, Schettler P, Drake DF et al (2013). A randomized controlled trial of the tumor necrosis factor antagonist infliximab for treatment-resistant depression: the role of baseline inflammatory biomarkers. JAMA Psychiatry 70: 31-41.

Reichenberg A, Yirmiya R, Schuld A, Kraus T, Haack M, Morag A et al (2001). Cytokine-associated emotional and cognitive disturbances in humans. Arch Gen Psychiatry 58: 445-452.

Schaller M, Miller GE, Gervais WM, Yager S, Chen E (2010). Mere visual perception of other people's disease symptoms facilitates a more aggressive immune response. Psychological Sci 21: 649-652.

Schiepers OJG, Wichers MC, Maes M (2005). Cytokines and major depression. Prog Neuropsychopharmacol Biol Psychiatry 29: 201-217.

Schultze-Florey CR, Martínez-Maza O, Magpantay L, Breen EC, Irwin MR, Gündel H et al (2012). When grief makes you sick: bereavement induced systemic inflammation is a question of genotype. Brain Behav Immun 26: 1066-1071.

Shankar A, McMunn A, Banks J, Steptoe A (2011). Loneliness, social isolation, and behavioral and biological health indicators in older adults. Health Psychology 30: 377.

Slavich GM, Cole SW (2013). The emerging field of human social genomics. Clin Psychological Sci 1: 331-348.

Slavich GM, Irwin MR (2014). From stress to inflammation and major depressive disorder: a social signal transduction theory of depression. Psychol Bull 140: 774

Slavich GM, Way BM, Eisenberger NI, Taylor SE (2010). Neural sensitivity to social rejection is associated with inflammatory responses to social stress. Proc Natl Acad Sci 107: 14817-14822.

Sloan EK, Capitanio JP, Tarara RP, Mendoza SP, Mason WA, Cole SW (2007). Social stress enhances sympathetic innervation of primate lymph nodes: mechanisms and implications for viral pathogenesis. J Neurosci 27: 8857-8865.

Slopen N, Kubzansky LD, McLaughlin KA, Koenen KC (2013). Childhood adversity and inflammatory processes in youth: a prospective study. Psychoneuroendocrinology 38: 188-200.

Steptoe A, Hamer M, Chida Y (2007). The effects of acute psychological stress on circulating inflammatory factors in humans: a review and meta-analysis. Brain Behav Immun 21: 901-912.

Stevenson RJ, Hodgson D, Oaten MJ, Moussavi M, Langberg R, Case Tl et al (2012). Disgust elevates core body temperature and up-regulates certain oral immune markers. Brain Behav Immun 26: 1160-1168.

Willette AA, Lubach GR, Coe CL (2007). Environmental context differentially affects behavioral, leukocyte, cortisol, and interleukin-6 responses to low doses of endotoxin in the rhesus monkey. Brain Behav Immun 21: 807-815.

Yamakawa K, Matsunaga M, Isowa T, Kimura K, Kasugai K, Yoneda M et al (2009). Transient responses of inflammatory cytokines in acute stress. Biol Psychol 82: 25-32.

Yee JR, Prendergast BJ (2010). Sex-specific social regulation of inflammatory responses and sickness behaviors. Brain Behav Immun 24: 942-951.

Zhang WN, Chang SH, Guo LY, Zhang KL, Wang J (2013). The neural correlates of reward-related processing in major depressive disorder: a meta-analysis of functional magnetic resonance imaging studies. J Affect Disord 151: 531-539. 